\title{
A Circulating Long Noncoding RNA Panel Serves as a Diagnostic Marker for Hepatocellular Carcinoma
}

\author{
Jinlan Huang, ${ }^{1,2}$ Yansong Zheng, ${ }^{3}$ Xialin Xiao, ${ }^{2,4}$ Can Liu, ${ }^{1,2}$ Jinpiao Lin, ${ }^{1,2}$ Sijia Zheng, \\ Bin Yang, ${ }^{1,2}$ and Qishui Ou $\mathbb{D}^{1,2}$ \\ ${ }^{1}$ Department of Laboratory Medicine, The First Affiliated Hospital of Fujian Medical University, Fuzhou, \\ Fujian Province 350005, China \\ ${ }^{2}$ Fujian Key Laboratory of Laboratory Medicine, Fuzhou, Fujian Province 350005, China \\ ${ }^{3}$ Department of Hepatobiliary Surgery, The First Affiliated Hospital of Fujian Medical University, Fuzhou, \\ Fujian Province 350005, China \\ ${ }^{4}$ Medical Technology and Engineering College, Fujian Medical University, Fuzhou, Fujian Province 350004, China
}

Correspondence should be addressed to Qishui Ou; ouqishui@fjmu.edu.cn

Received 5 November 2019; Revised 26 June 2020; Accepted 30 June 2020; Published 14 July 2020

Academic Editor: Roberta Rizzo

Copyright (C) 2020 Jinlan Huang et al. This is an open access article distributed under the Creative Commons Attribution License, which permits unrestricted use, distribution, and reproduction in any medium, provided the original work is properly cited.

\begin{abstract}
Background. Circulating long noncoding RNAs (lncRNAs) have been demonstrated to serve as diagnostic biomarkers for various cancers. We aimed to elucidate the diagnostic efficacy of eight serum lncRNAs HULC, MALAT1, Linc00152, PTENP1, PTTG3P, SPRY4-IT1, UBE2CP3, and UCA1 and their combinations for the diagnosis of hepatocellular carcinoma (HCC). Methods. A total of 129 patients with HCC, 49 patients with liver cirrhosis, 27 patients with chronic hepatitis B, and 93 healthy controls were enrolled in this study. The levels of serum lncRNAs were assessed by quantitative real-time polymerase chain reaction. The correlations between serum lncRNAs and clinicopathological characteristics were further analyzed. The receiver operating characteristic (ROC) curve and area under curve (AUC) were utilized to estimate the diagnostic capacity of serum lncRNAs and their combination with AFP for HCC. A logistic regression model was performed to establish a multiple-lncRNA panel. Results. The levels of serum HULC, MALAT1, Linc00152, PTTG3P, SPRY4-IT1, UBE2CP3, and UCA1 were significantly higher in HCC patients than in patients with benign liver diseases and healthy controls, whereas serum PTENP1 was significantly decreased in HCC patients compared with healthy participants. Positive correlations between serum Linc00152 and GGT, serum PTTG3P and GGT, and serum SPRY4-IT1 and ALT were noted in HCC patients. ROC analysis revealed that all these lncRNAs had a significantly predictive value for HCC except for PTENP1. The best performance of single lncRNA was obtained by Linc00152 with an AUC of 0.877. When combined with AFP, the combination of Linc00152 and AFP gained the highest accuracy, yielding an AUC of 0.906. Through logistic regression analysis, the panel consisting of serum linc00152, UCA1, and AFP provided the greatest predictive ability, obtaining an AUC of 0.912 with $82.9 \%$ sensitivity and $88.2 \%$ specificity. Conclusion. The panel of serum Linc00152, UCA1, and AFP demonstrates a novel and noninvasive biomarker with relatively high sensitivity and specificity for HCC diagnosis.
\end{abstract}

\section{Introduction}

Hepatocellular carcinoma (HCC) is one of the most common cancers globally with relatively high morbidity and mortality [1]. Despite great progresses made in HCC therapy in recent years, the prognosis of HCC patients still remains poor due to being diagnosed at an advanced stage and high rate of recurrence [2]. Currently, histological examination of the tumor tissue serves as the standard procedure for definitive diagnosis of HCC, while imaging examinations including ultrasound and computer tomography are the common supplement procedures [3]. However, 
TABLE 1: Sequences of primers used in this study.

\begin{tabular}{lcc}
\hline qRT-PCR primers & Sequences \\
\hline HULC & Sense & ATCTGCAAGCCAGGAAGAGTC \\
& Antisense & CTTGCTTGATGCTTTGGTCTGT \\
MALAT1 & Sense & AAAGCAAGGTCTCCCCACAAG \\
& Antisense & GGTCTGTGCTAGATCAAAAGGCA \\
Linc00152 & Sense & GACTGGATGGTCGCTGCTTT \\
& Antisense & CCCAGGAACTGTGCTGTGAA \\
PTENP1 & Sense & CATTCTTGCATGTATTTGGGTTAGG \\
& Antisense & GGTATATGGTCCAGAGTCCAGC \\
PTTG3P & Sense & GGGGTCTGGACCTTCAATCAA \\
& Antisense & GCTTTAGGTAAGGATGTGGGA \\
SPRY4-IT1 & Sense & GTTTTTGCTGAGCTGGTGGTT \\
& Antisense & ATGGCTCCACTGGGCATATT \\
UBE2CP3 & Sense & AAGTGGTCTGCCCTGTATGATG \\
& Antisense & GAGCTATCAATGTTGGGTTTGC \\
UCA1 & Sense & TGCACCCTAGACCCGAAACT \\
& Antisense & CAAGTGTGACCAGGGACTGC \\
GAPDH & Sense & GGGAAACTGTGGCGTGAT \\
& Antisense & GAGTGGGTGTCGCTGTTGA \\
\hline
\end{tabular}

histopathology may cause hemorrhage and tissue damage, and imaging examinations are expensive and lack adequate sensitivity and specificity. For serum biomarkers, alpha fetoprotein (AFP) is one of the most commonly used biomarkers for HCC screening and diagnosis clinically [4]. Nevertheless, the sensitivity and specificity of AFP are unsatisfactory, especially for patients with early-stage HCC [5]. Therefore, it is of great value to develop novel and noninvasive biomarkers with improved sensitivity and specificity for HCC diagnosis and prognosis.

Accumulating evidence highlights the critical roles of long noncoding RNA (lncRNA) in the initiation and progression of HCC. The lncRNAs, which are defined as noncoding RNA molecules $>200$ nucleotides in length, have been confirmed to play crucial roles in various biological processes involved in hepatocarcinogenesis, including cell growth, angiogenesis, and metastasis [6]. In addition, previous reports have confirmed the stable presence of lncRNAs in various body fluids such as plasma, serum, urine, gastric juice, and saliva [7]. Several circulating lncRNAs have been implied to be promising markers with high accuracies and efficiencies for the diagnosis and prognosis of HCC [8-10]. Examples include HULC, MALAT1, Linc00152, UCA1, SPRY4-IT1, and UBE2CP3 $[9,11]$. However, the diagnostic value of many lncRNAs for HCC reported by different researchers remains controversial and needs further investigation. Moreover, the diagnostic efficacy of only a small number of circulating lncRNAs has been confirmed. The majority of circulating lncRNAs involved in HCC still remains largely unclear.

Previous studies have reported that serum PTENP1 can distinguish patients with gastric cancer or clear cell renal cell carcinoma from healthy controls $[12,13]$ and PTENP1 func- tions as a vital tumor suppressor for HCC [14]. Moreover, we previously found PTTG3P plays an important role in HCC progression and can be detectable in the serum of HCC patients [15]. Therefore, in this study, we initially enrolled PTENP1, PTTG3P, and other six lncRNAs (HULC, MALAT1, Linc00152, SPRY4-IT1, UBE2CP3, and UCA1) which have been already reported to be promising serum markers for HCC $[9,11,15]$. This study is aimed at evaluating the serum levels of these eight lncRNAs among patients with HCC, chronic hepatitis B (CHB), and liver cirrhosis (LC) and healthy participants and at assessing the diagnostic values of these lncRNAs and their combination with AFP, thus to identify a panel of circulating lncRNAs as a tool for the diagnosis of HCC.

\section{Materials and Methods}

2.1. Patients. A total of 298 individuals were enrolled in the study (129 patients with HCC, 27 patients with CHB, 49 patients with LC, and 93 healthy controls). All patients were recruited from the First Affiliated Hospital of Fujian Medical University between September 2017 and May 2019. The patients with HCC were histologically confirmed. Patients with $\mathrm{CHB}$ were diagnosed according to the Clinical Practice Guidelines on the management of hepatitis B virus infection of EASL in 2017. Patients with LC were verified according to the American Association for the Study of Liver Diseases Practice Guidelines. Clinical characteristics of patients were obtained from the medical records retrospectively, including age, gender, HBV surface antigen (HBsAg), tumor number, and tumor size. As a healthy control, 93 individuals who performed their annual health check at the hospital and did not have any liver diseases or other cancerous diseases were recruited. Written informed consent was obtained from each participant, and the study was approved by the Ethics Committee of the First Affiliated Hospital of Fujian Medical University (2018[048]).

2.2. Sample Collection. Peripheral blood samples were collected in a separate vacuum tube from all participants before surgery, pharmacological intervention, or chemotherapy. The serum was isolated by centrifugation at 3,000 rpm for $10 \mathrm{~min}$. After that, the isolated serum was immediately transferred and then stored at $-80^{\circ} \mathrm{C}$ for further analysis.

2.3. RNA Isolation and Quantitative Real-Time PCR ( $q R T$ $P C R$ ). Total RNA was extracted from serum samples using a Hipure Liquid RNA Kit (Cat\# R4163-03, Magen, Guangzhou, China). The RNA quantity and purity were evaluated via the NanoDrop One spectrophotometer (Thermo Scientific, Wilmington, DE, USA). The purified RNA was reversely transcribed into cDNA using the M-MLV Reverse Transcriptase (Cat\# M1701, Promega, Madison WI, USA). Then, the levels of lncRNAs were assessed by qRT-PCR using TB Green $^{\mathrm{TM}}$ Premix Ex Taq (Cat\# RR420A, Takara, Dalian, China) which was performed on the QuantStudio Real Time PCR system (Applied Biosystems, Foster City, CA, USA). All reactions were performed with the following conditions: $95^{\circ} \mathrm{C}$ for 30 seconds, 45 cycles of $95^{\circ} \mathrm{C}$ for 5 seconds, and 
TABLE 2: Demographics of healthy controls and patient with CHB, LC, and HCC.

\begin{tabular}{lcccc}
\hline Features & HC & CHB & LC & HCC \\
\hline Number & 93 & 27 & 49 & 129 \\
Age & $55(21-79)$ & $52(33-71)$ & $58(35-87)$ & $59(23-88)$ \\
Male/female & $69 / 24$ & $21 / 6$ & $32 / 17$ & $111 / 18$ \\
AFP $(\mathrm{ng} / \mathrm{mL})$ & $2.22(0.68-6.70)$ & $2.62(0.95-569.0)$ & $4.03(0.61-759.0)$ & $38.50(0.61-22484.0)$ \\
GGT (U/L) & $16(7-59)$ & $36(8-209)$ & $64.5(9-759)$ & $80(13-2000)$ \\
ALT (U/L) & $18(9-46)$ & $28(12-2112)$ & $29.5(8-1688)$ & $37(7-1198)$ \\
AST (U/L) & $20(14-38)$ & $25(18-1856)$ & $54(16-639)$ & $41(17-1474)$ \\
TP (g/L) & $73.7(65.1-81.8)$ & $71.1(59.1-77)$ & $63.7(48.6-78.2)$ & $68.6(43.4-84.8)$ \\
ALB (g/L) & $45.5(40.5-50.5)$ & $45.1(28-49.2)$ & $28.8(22.5-28.6)$ & $40.1(21.4-48.9)$ \\
\hline
\end{tabular}

HC: healthy control; HCC: hepatocellular carcinoma; CHB: chronic hepatitis B; LC: liver cirrhosis; AFP: alpha fetoprotein; GGT: gamma-glutamyl transpeptidase; ALT: alanine aminotransferase; AST: aspartic transaminase; TP: total protein; ALB: albumin.

$60^{\circ} \mathrm{C}$ for 30 seconds. The specificity of the PCR products was ensured by melting curve analysis following each reaction. The relative expression of each IncRNA was determined using the $2-\Delta \Delta \mathrm{Ct}$ method with GAPDH as the endogenous control for data normalization. The primers used are listed in Table 1.

2.4. Clinical Chemistry Analysis and Serum AFP Detection. Serum alanine aminotransferase (ALT), aspartic transaminase (AST), gamma-glutamyl transpeptidase (GGT), total protein (TP), and albumin (ALB) were measured on the ADVIA 2400 clinical chemistry analyzer (Siemens, Germany). Serum AFP was detected on the Roche Cobas analyzer (Roche Diagnostics, Mannheim, Germany).

2.5. Statistical Analysis. Statistical analysis was carried out by SPSS 23.0 software (SPSS Inc., Chicago, IL, USA) and GraphPad prism 7.0 software (GraphPad Software, Inc., La Jolla, CA, USA). Specifically, the Kolmogorov-Smirnov test was performed to analyze the normal distribution of the variables. If the variables were normally distributed, they were presented as the mean \pm standard deviations (SD) and analyzed by two-tailed Student's $t$-test or one-way ANOVA. Besides, for nonparametric data, the Mann-Whitney $U$ test was used for comparisons between groups. Pearson's correlation coefficient test was applied to judge the correlations between two variables with normal distribution while Spearman's correlation coefficient test was used for variables with abnormal distribution. Values of the area under curve (AUC) obtained from receiver operating characteristic (ROC) curve were performed to evaluate the diagnostic performance of HULC, PTENP1, MALAT1, Linc00152, PTTG3P, UBE2CP3, SPRY4-IT1, UCA1, AFP, and their combinations. The multiIncRNA panel was established by logistic regression analysis. A two-tailed $P$ value of $<0.05$ was considered statistically significant.

\section{Results}

3.1. Patient Characteristics. A total of 298 individuals including 129 patients with HCC, 27 patients with $\mathrm{CHB}, 49$ patients with LC, and 93 healthy controls were enrolled into this study. Table 2 lists the detailed demographic information of all participants. The median age was 59 years (range, 23-88 years) for patients with HCC, 58 years (range, 35-87 years) for patients with LC, 52 years (range, 33-71 years) for patients with $\mathrm{CHB}$, and 55 years (21-79 years) for healthy controls. The HCC cohort consisted of 111 males and 18 females. For patients with LC, 32 patients were male and 17 were female. The $\mathrm{CHB}$ group contained 21 males and 6 females. As for healthy controls, 69 participants were male and 24 were female.

3.2. Serum Levels of IncRNAs in Patients with HCC, LC, and $\mathrm{CHB}$ and Healthy Controls. As shown in Figure 1, significantly high levels of lncRNA HULC, MALAT1, Linc00152, PTTG3P, SPRY4-IT1, UBE2CP3, and UCA1 were observed in patients with HCC when compared with healthy controls, CHB patients, or LC patients. Serum PTENP1 was markedly decreased in HCC patients than in healthy controls, whereas there was no significant difference with respect to serum PTENP1 between HCC patients and CHB patients or LC patients (Figure 1(d)). Elevated levels of HULC, Linc00152, SPRY4-IT1, UBE2CP3, and UCA1 and the declined level of PTENP1 were observed in patients with LC relatively to healthy controls (Figure 1). Surprisingly, the levels of all eight lncRNAs showed no significance between the LC and CHB groups (Figure 1). Patients with $\mathrm{CHB}$ had higher levels of MALAT1, SPRY4-IT1, UBE2CP3, and UCA1 than healthy controls while the levels of other four lncRNAs exhibited no statistical difference (Figure 1).

3.3. Correlations between Serum IncRNAs and Clinicopathological Characteristics. We further investigated the associations between the serum levels of eight lncRNAs and clinicopathological features in patients with HCC. Our results showed that serum Linc00152 were positively correlated with GGT $(R=0.245, P=0.005$; Table 3, Figures 2(a) and 2(b)). Positive correlations were also observed between PTTG3P and GGT $(R=0.225, P=0.01$; Table 4, Figures 2(c) and 2(d)). Furthermore, HCC patients with a lower level of serum PTENP1 tended to have a higher level of AFP $(P=0.064$, Table 4$)$. Serum levels of serum SPRY4-IT1 were positively associated with ALT $(R=0.173$, $P=0.05$; Table 4, Figures 2(e) and 2(f)). HCC patients older 


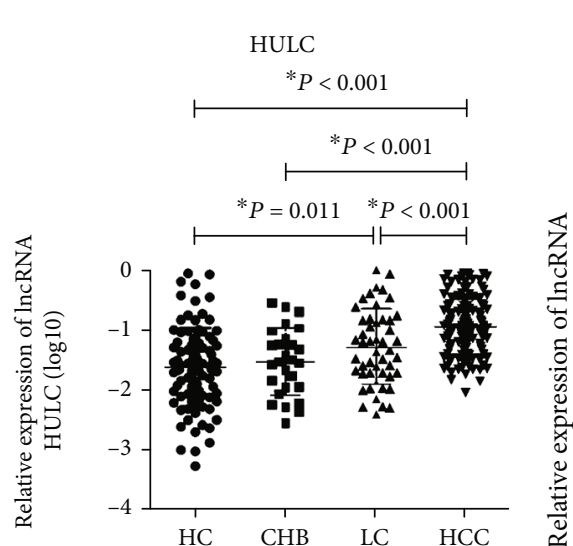

(a)

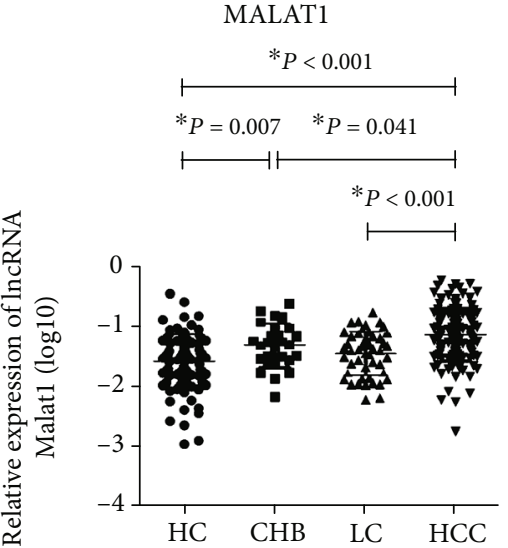

(b)

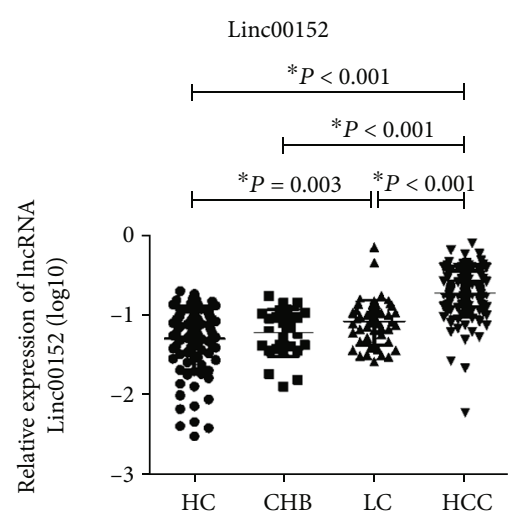

(c)

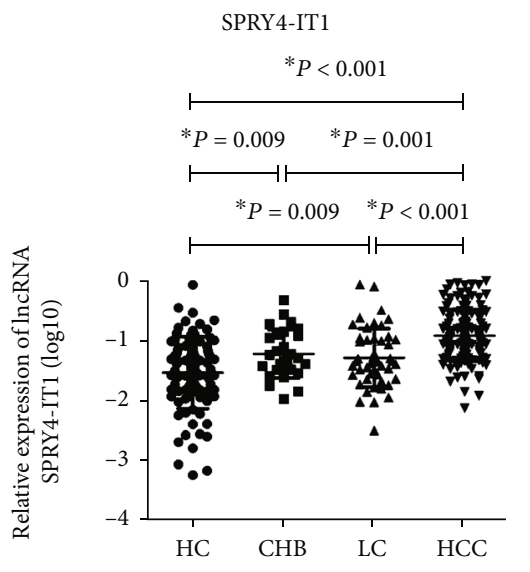

(f)

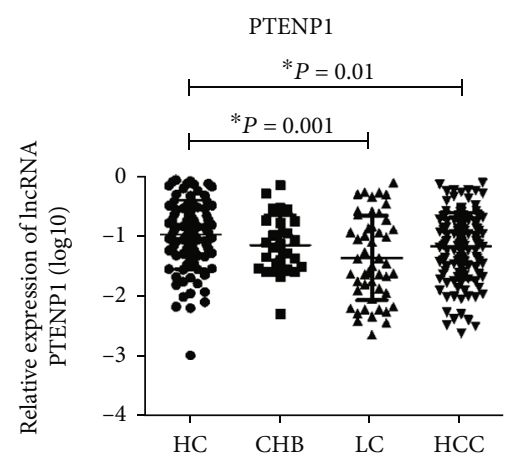

(d)

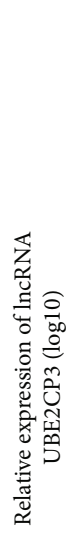

UBE2CP3
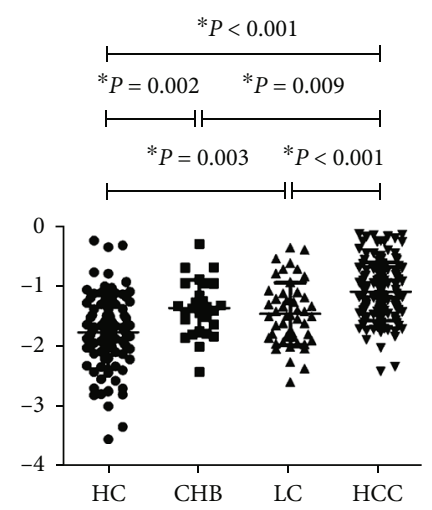

(g)

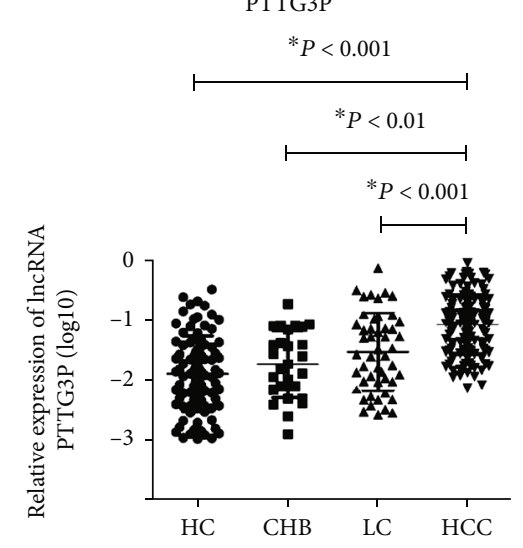

(e)

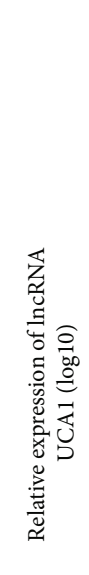

UCA1
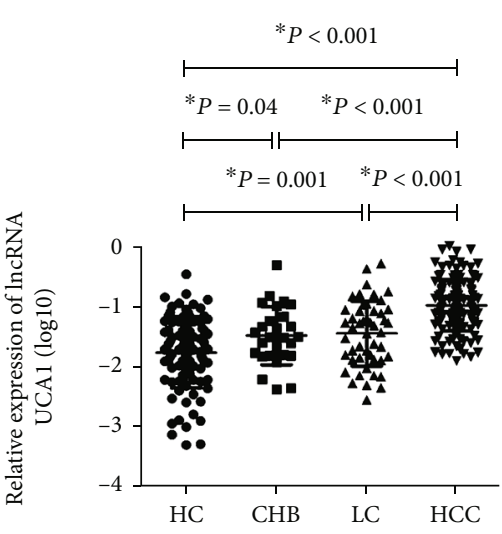

(h)

FIGURE 1: The levels of eight serum lncRNAs in patients with HCC, CHB, LC and healthy controls. The relative expression levels of lncRNAs HULC (a), MALAT1 (b), Linc00152 (c), PTENP1 (d), PTTG3P (e), SPRY4-IT1 (f), UBE2CP3 (g), and UCA1 (h) in the serum of patients with HCC $(n=129)$, CHB $(n=27)$, LC $(n=49)$, and healthy controls $(n=93)$ by qRT-PCR. Circulating lncRNA expressions were calculated using $2-\Delta \Delta \mathrm{Ct}$ method. GAPDH was used as a normalization control. Data of relative expression of serum lncRNAs fitted normal distribution after $\log 10$ transformation, and thus, the independent $t$-test was applied for comparisons between groups.

than 55 years tended to have a lower level of SPRY4-IT1 and UCA1 (Tables 4 and 5, Figures 2(g) and 2(h)). However, we did not observe any associations between serum lncRNA and gender, HBsAg, tumor size, tumor number, AST, TP, and ALB.
3.4. Diagnosis Efficacy of Each Serum lncRNA in Patients with HCC. Figures 3(a) and 3(b) show the diagnostic value (ROC curves) of $8 \operatorname{lncRNAs}$ in patients with HCC. When HCC patients were tested against healthy controls, the AUC was 0.796 (0.734-0.858) for HULC, 0.768 (0.706-0.830) for 
TABLE 3: Correlation between serum level of lncRNAs HULC, MALAT1, and Linc00152 and clinicopathological characteristics in 129 HCC patients.

\begin{tabular}{|c|c|c|c|c|c|c|c|c|c|}
\hline \multirow{2}{*}{ Features } & \multicolumn{3}{|c|}{ HULC } & \multicolumn{3}{|c|}{ MALAT1 } & \multicolumn{3}{|c|}{ Linc00152 } \\
\hline & Low $(n=65)$ & High $(n=64)$ & $P$ & Low $(n=65)$ & High $(n=64)$ & $P$ & Low $(n=65)$ & High $(n=64)$ & $P$ \\
\hline Gender & & & 0.327 & & & 0.327 & & & 0.636 \\
\hline Male & 54 & 57 & & 54 & 57 & & 55 & 56 & \\
\hline Female & 11 & 7 & & 11 & 7 & & 10 & 8 & \\
\hline Age & & & 0.333 & & & 0.333 & & & 0.801 \\
\hline$\leq 55$ & 24 & 29 & & 24 & 29 & & 26 & 27 & \\
\hline$>55$ & 41 & 35 & & 41 & 35 & & 39 & 37 & \\
\hline HBsAg & & & 0.270 & & & 0.314 & & & 0.796 \\
\hline Positive & 53 & 47 & & 48 & 52 & & 51 & 49 & \\
\hline Negative & 12 & 17 & & 17 & 12 & & 14 & 15 & \\
\hline AFP level & & & 0.781 & & & 0.323 & & & 0.127 \\
\hline$\leq 20 \mathrm{ng} / \mathrm{mL}$ & 29 & 27 & & 31 & 25 & & 33 & 41 & \\
\hline$>20 \mathrm{ng} / \mathrm{mL}$ & 36 & 37 & & 34 & 39 & & 32 & 23 & \\
\hline Tumor size & & & 0.897 & & & 0.530 & & & 0.315 \\
\hline$\leq 3 \mathrm{~cm}$ & 21 & 20 & & 19 & 22 & & 18 & 23 & \\
\hline$>3 \mathrm{~cm}$ & 44 & 44 & & 46 & 42 & & 47 & 41 & \\
\hline $\begin{array}{l}\text { Tumor } \\
\text { number }\end{array}$ & & & 0.611 & & & 0.668 & & & 0.611 \\
\hline Single & 55 & 52 & & 53 & 54 & & 55 & 52 & \\
\hline Multiple & 10 & 12 & & 12 & 10 & & 10 & 12 & \\
\hline GGT (U/L) & $80(14-2000)$ & $77(13-1034)$ & 0.944 & $80(13-2000)$ & $84(14-1034)$ & 0.265 & $61(13-697)$ & $98(15-2000)$ & $0.012^{*}$ \\
\hline $\operatorname{ALT}(\mathrm{U} / \mathrm{L})$ & $40(7-451)$ & $37(12-1198)$ & 0.944 & $37(7-621)$ & $37.5(12-1198)$ & 0.929 & $34(7-668)$ & $40(12-1198)$ & 0.378 \\
\hline AST (U/L) & $47(17-577)$ & $41(17-1474)$ & 0.901 & $38(18-577)$ & $51(17-1474)$ & 0.142 & $36(17-577)$ & $48(17-1474)$ & 0.077 \\
\hline $\mathrm{TP}(\mathrm{g} / \mathrm{L})$ & $69.5(56.0-80.6)$ & $68.3(43.4-84.8)$ & 0.376 & $68.8(55.3-84.8)$ & $68.3(43.4-84.2)$ & 0.232 & $68.4(46.8-79)$ & $68.7(43.4-84.8)$ & 0.644 \\
\hline $\operatorname{ALB}(g / L)$ & $40.3(23.9-48.9)$ & $39.3(21.4-48.6)$ & 0.564 & $40.3(23.8-48.9)$ & $39.2(21.4-47.7)$ & 0.170 & $40.0(23.9-47.2)$ & $40.2(21.4-48.9)$ & 0.888 \\
\hline
\end{tabular}

${ }^{*} P<0.05$. AFP: alpha fetoprotein; GGT: gamma-glutamyl transpeptidase; ALT: alanine aminotransferase; AST: aspartic transaminase; TP: total protein; ALB: albumin.

MALAT1, 0.895 (0.854-0.936) for Linc00152, 0.602 (0.5260.678) for PTENP1, 0.785 (0.723-0.847) for PTTG3P, 0.808 $(0.750-0.866)$ for SPRY4-IT1, $0.812(0.754-0.870)$ for UBE2CP3, 0.858 (0.810-0.907) for UCA1, and 0.862 (0.8150.909 ) for AFP, respectively (Figure 3(a) and Table 6). Among these markers, Linc00152 demonstrated the highest accuracy in predicting HCC from healthy participants with $78.3 \%$ sensitivity and $89.2 \%$ specificity (Figure $3(\mathrm{a}$ ) and Table 6).

We further explored whether the relative levels of the 8 lncRNAs could discriminate HCC patients from patients with $\mathrm{CHB}$ and LC. As shown in Figure 3(b) and Table 6, ROC analysis showed that AFP (AUC: 0.811; 95\% CI: 0.761-0.861), HULC (AUC: 0.756; 95\% CI: 0.702-0.810), MALAT1 (AUC: 0.733; 95\% CI: 0.676-0.790), Linc00152 (AUC: 0.877; 95\% CI: 0.835-0.918), PTTG3P (AUC: 0.768; 95\% CI: 0.715-0.820), SPRY4-IT1 (AUC: 0.768; 95\% CI: 0.715-0.821), UBE2CP3 (AUC: 0.756; 95\% CI: 0.702-0.810), and UCA1 (AUC: 0.809 ; 95\% CI: 0.761-0.857) had a significantly predictive value for distinguishing HCC patients from CHB and LC patients and HC. However, PTENP1 (AUC:
0.530; 95\% CI: 0.465-0.596) did not show any diagnosis efficacy to predicting HCC from CHB patients, LC patients, and healthy controls $(P>0.05$, Figure 3(b) and Table 6). The best diagnostic value was obtained with Linc00152 with $81.4 \%$ sensitivity and $82.8 \%$ specificity (Figure 3 (b) and Table 6 ).

3.5. Diagnosis Efficacy of the Combination of Serum IncRNAs with AFP and Establishment of Diagnostic Panel. Given the fact that AFP is one of the most common markers used for HCC screening and diagnosis, we also evaluated the combinations of these seven lncRNAs with AFP for the diagnosis of HCC. The AUC value, 95\% CI, sensitivity, and specificity for the combinations of serum lncRNAs and AFP to discriminate HCC from CHB patients, LC patients, and healthy individuals are listed in Table 7. The AUC values of the combination of seven lncRNAs with AFP to predicting HCC were higher than that of AFP alone (Figure 3(c) and Table 7). The combination of Linc00152 and AFP gained the highest accuracy, yielding an AUC of 0.906 (95\% CI: $0.870-0.942$ ) with $85.3 \%$ sensitivity and $83.4 \%$ specificity (Figure 3(c) and Table 7). 


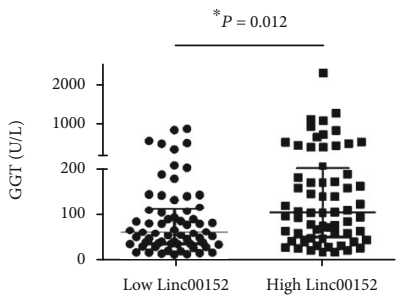

(a)

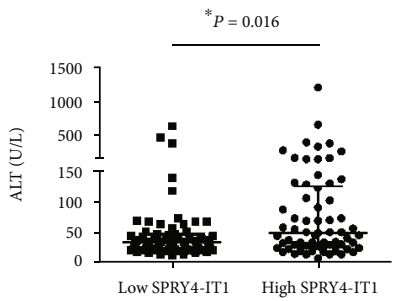

(e)

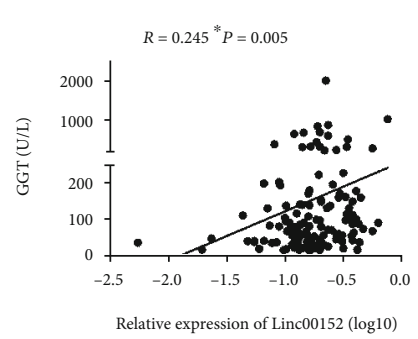

(b)

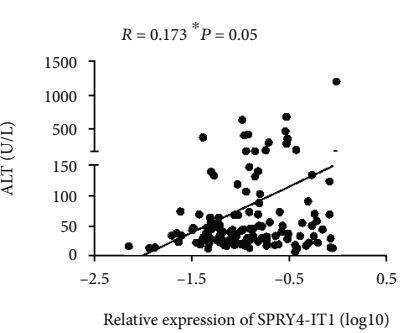

(f)

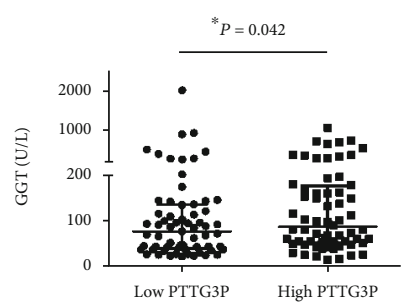

(c)

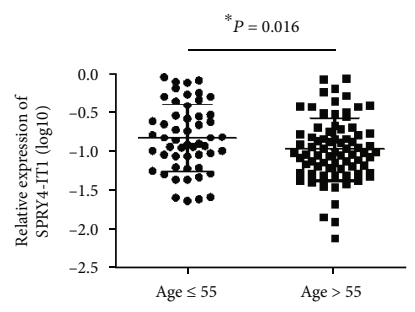

(g)

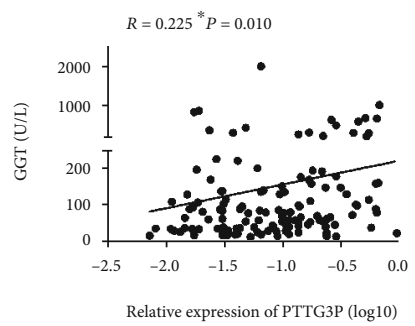

(d)

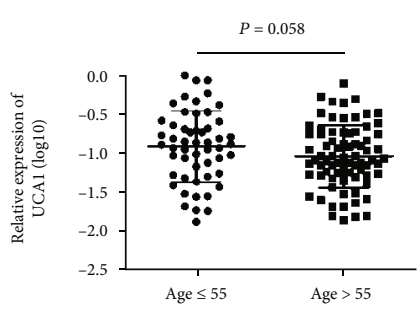

(h)

FIGURE 2: Correlation between serum lncRNAs and clinicopathological characteristics. (a) The level of GGT in patients with low level of Linc00152 $(n=65)$ was significantly lower than that in patients with high level of Linc00152 $(n=64)$. Statistical differences were analyzed by the independent $t$-test. (b) The relative expression of serum Linc00152 was positively correlated with GGT in 129 HCC patients. The linear correlation was calculated by Pearson's correlation analysis. (c) Patients with high expression of serum PTTG3P ( $n=64)$ had a higher level of GGT than those with low expression of serum PTTG3P $(n=65)$. (d) Pearson's correlation analysis revealed a positive correlation between the serum levels of PTTG3P and GGT. (e) High levels of ALT were observed in patients with high SPRY4-IT1 relatively to those with low SPRY4-IT1. (f) The relative level of SPRY4-IT1 was positively correlated to ALT. Patients older than 55 years tended to have lower levels of SPRY4-IT1 (g) and UCA1 (h).

Based on these data, a stepwise regression model was established to get the strongest panel for predicting HCC. Finally, two lncRNAs (Linc00152 and UCA1) and AFP were enrolled. The final formula was listed as follows: $4.528+$ $3.784 \times$ Linc $00152+1.189 \times \mathrm{UCA} 1+0.005 \times$ AFP. The panel of Linc00152, UCA1, and AFP provided the greatest predictive ability, obtaining an AUC of 0.912 (95\% CI: 0.878-0.945) with $82.9 \%$ sensitivity and $88.2 \%$ specificity (Figure $3(\mathrm{~d})$ and Table 7).

\section{Discussion}

HCC is an aggressive tumor with high rates of recurrence and metastasis, which leads to the poor prognosis. Therefore, it is of great value to find an effective biomarker for the early diagnosis and therapy of HCC. Previous studies have confirmed that lncRNAs are detectable and also stable in serum of cancer patients, making it possible to be utilized as diagnostic biomarkers for various cancers [16, 17]. Currently, several lncRNAs have been reported to be dysregulated in the serum or plasma of HCC patients. For instance, HULC is highly expressed in tumor tissues as well as plasma of HCC patients, and plasma HULC achieves a fine diagnostic accuracy in predicting HCC [18]. However, clinical use of circulating lncRNAs for HCC still remains controversial due to inconsistent results reported by different researchers and some technical issues, such as using single or multiple lncRNAs.
In this study, we selected eight lncRNAs (HULC, MALAT1, Linc00152, PTENP1, PTTG3P, SPRY4-IT1, UBE2CP3, and UCA1) from previously published HCCrelated studies $[11,14,15,18-20]$ and assessed their expressions in serum of 129 HCC patients, 49 patients with LC, 27 patients with $\mathrm{CHB}$, and 93 healthy controls. Our data revealed that seven IncRNAs (HULC, MALAT1, Linc00152, PTTG3P, SPRY4-IT1, UBE2CP3, and UCA1) were markedly high expressed in serum of HCC patients compared with healthy controls, which are similar to some previous studies $[11,18,21-23]$. These results lead to an indication that these lncRNAs might be useful candidates for the screening and diagnosis of HCC. The elevation of circulating lncRNAs may result from the increased release of nucleic acids from necrosis tumor cells or increased secretion of extracellular vesicles by tumor cells into the blood $[17,24]$. PTENP1 is a reported tumor suppressor for HCC [14]. Here, we revealed that serum PTENP1 was significantly downregulated in HCC patients. Moreover, MALAT1, SPRY4-IT1, UBE2CP3, and UCA1 were upregulated in $\mathrm{CHB}$ patients, indicating they may be involved in the early period of liver injury. The increased level of all these lncRNAs was observed in LC patients except for MALAT1. Given the fact that LC increases the risk of HCC with an annual incidence between 2 and 4\% [3], these seven lncRNAs might play important roles in the early stage of HCC initiation. Intriguingly, plasma MALAT1 is reported to be upregulated in $\mathrm{CHB}$ patients and also LC patients [25]. In our study, significant elevation of serum MALAT1 is noted in patients with $\mathrm{CHB}$ 
TABLE 4: Correlation between serum level of lncRNAs PTENP1, PTTG3P, and SPRY4-IT1 and clinicopathological characteristics in 129 HCC patients.

\begin{tabular}{|c|c|c|c|c|c|c|c|c|c|}
\hline \multirow{2}{*}{ Features } & \multicolumn{3}{|c|}{ PTENP1 } & \multicolumn{3}{|c|}{ PTTG3P } & \multicolumn{3}{|c|}{ SPRY4-IT1 } \\
\hline & Low $(n=65)$ & High $(n=64)$ & $P$ & Low $(n=65)$ & $\operatorname{High}(n=64)$ & $P$ & Low $(n=65)$ & $\operatorname{High}(n=64)$ & $P$ \\
\hline Gender & & & 0.972 & & & 0.136 & & & 0.636 \\
\hline Male & 56 & 55 & & 53 & 58 & & 55 & 56 & \\
\hline Female & 9 & 9 & & 12 & 6 & & 10 & 8 & \\
\hline Age & & & 0.124 & & & 0.185 & & & $0.016^{*}$ \\
\hline$\leq 55$ & 31 & 22 & & 23 & 30 & & 20 & 33 & \\
\hline$>55$ & 34 & 42 & & 42 & 34 & & 45 & 31 & \\
\hline HBsAg & & & 0.558 & & & 0.870 & & & 0.496 \\
\hline Positive & 49 & 51 & & 50 & 50 & & 52 & 48 & \\
\hline Negative & 16 & 13 & & 15 & 14 & & 13 & 16 & \\
\hline AFP level & & & 0.064 & & & 0.089 & & & 0.858 \\
\hline$\leq 20 \mathrm{ng} / \mathrm{mL}$ & 23 & 33 & & 33 & 23 & & 28 & 27 & \\
\hline$>20 \mathrm{ng} / \mathrm{mL}$ & 42 & 31 & & 32 & 41 & & 36 & 37 & \\
\hline Tumor size & & & 0.897 & & & 0.612 & & & 0.530 \\
\hline$\leq 3 \mathrm{~cm}$ & 21 & 20 & & 22 & 19 & & 19 & 22 & \\
\hline$>3 \mathrm{~cm}$ & 44 & 44 & & 43 & 45 & & 46 & 42 & \\
\hline $\begin{array}{l}\text { Tumor } \\
\text { number }\end{array}$ & & & 0.968 & & & 0.329 & & & 0.329 \\
\hline Single & 54 & 53 & & 56 & 51 & & 56 & 51 & \\
\hline Multiple & 11 & 11 & & 9 & 13 & & 9 & 13 & \\
\hline GGT (U/L) & $90(14-1034)$ & $79.5(13-2000)$ & 0.611 & $70(13-2000)$ & $85.5(14-1034)$ & $0.042^{*}$ & $86(13-2000)$ & $65(15-710)$ & 0.517 \\
\hline ALT (U/L) & $38(12-1198)$ & $35(7-402)$ & 0.202 & $33(7-402)$ & $42.5(12-1198)$ & 0.316 & $33.5(12-621)$ & $49(7-1198)$ & $0.016^{*}$ \\
\hline AST (U/L) & $50(17-1474)$ & $38(17-577)$ & 0.306 & $37(18-577)$ & $52(17-1474)$ & 0.095 & $36.5(17-412)$ & $50(18-1474)$ & 0.097 \\
\hline $\mathrm{TP}(\mathrm{g} / \mathrm{L})$ & $68.2(43.4-84.8)$ & $69.1(55.5-80.6)$ & 0.504 & $68.6(55.5-80.6)$ & $68.6(43.4-84.8)$ & 0.983 & $68.6(46.8-80.6)$ & $68.7(43.4-84.8)$ & 0.243 \\
\hline $\operatorname{ALB}(g / L)$ & $39.4(21.4-48.6)$ & $40.5(23.8-48.9)$ & 0.301 & $40.3(23.8-48.9)$ & $39.5(21.4-48.6)$ & 0.539 & $39.6(21.4-48.9)$ & $40.2(23.6-48.6)$ & 0.468 \\
\hline
\end{tabular}

${ }^{*} P<0.05$. AFP: alpha fetoprotein; GGT: gamma-glutamyl transpeptidase; ALT: alanine aminotransferase; AST: aspartic transaminase; TP: total protein; ALB: albumin.

while only an increasing trend is observed in LC patients. The limited sample size may be possible to be blamed. As for PTTG3P, our previous study has demonstrated the vital roles of PTTG3P in promoting cell growth and metastasis in HCC [15]. Here, we provide the first evidence that the serum level of PTTG3P is markedly high in HCC patients relatively to patients with LC and CHB and healthy controls. This finding provides a basis for the application of PTTG3P as a novel diagnostic biomarker of HCC.

Next, we also investigated the correlations between these circulating lncRNAs and clinicopathological features. A significant association between serum Linc00152 and GGT as well as serum PTTG3P and GGT was demonstrated in patients with HCC. GGT is an enzyme which can be induced by intrahepatic obstruction or directly synthesized by liver cancer cells in HCC [26]. The diagnostic value of GGT has been deeply studied, and elevated GGT has been demonstrated to predict poor survival for HCC [27]. Accordingly, serum linc00152 and PTTG3P might be associated with the prognosis for HCC patients. Our data also revealed an inverse trend between serum PTENP1 and AFP. Further- more, we found that serum SPRY4-IT1 was positively correlated with ALT. Clinically, ALT is a useful indicator of liver injury. When liver cells are damaged, ALT will leak out from the injured cells into the blood, resulting in an increased serum ALT level [26]. Thus, it is suggested that serum SPRY4-IT1 may also be induced by liver injury. Besides, serum SPRY4-IT1 and UCA1 tended to decrease along with age in patients with HCC. In another word, younger patients tended to have higher levels of serum SPRY4-IT1 and UCA1. The same tendency of UCA1 along with age is reported by Kamel and his colleagues [28]. Some researchers found that young HCC patients have a larger tumor size and more advanced tumor stage at the time of diagnosis and thus show a worse prognosis than elder ones [29]. Therefore, we speculate that high serum levels of SPRY4-IT1 and UCA1 may serve as unfavorable prognostic factors for HCC.

To further investigate the diagnostic values of these lncRNAs, ROC curve analysis was performed. The AUC of the single lncRNA in our study is similar to some previous studies $[9,18]$. We firstly investigate the diagnostic values of serum PTENP1 and PTTG3P for HCC. Serum PTTG3P 
TABLE 5: Correlation between serum level of lncRNAs UBE2CP3 and UCA1 and clinicopathological characteristics in 129 HCC patients.

\begin{tabular}{|c|c|c|c|c|c|c|}
\hline \multirow{2}{*}{ Features } & \multicolumn{3}{|c|}{ UBE2CP3 } & \multicolumn{3}{|c|}{ UCA1 } \\
\hline & Low $(n=65)$ & High $(n=64)$ & $P$ & Low $(n=64)$ & High $(n=65)$ & $P$ \\
\hline Gender & & & 0.636 & & & 0.972 \\
\hline Male & 55 & 56 & & 55 & 56 & \\
\hline Female & 10 & 8 & & 9 & 9 & \\
\hline Age & & & 0.092 & & & 0.058 \\
\hline$\leq 55$ & 22 & 31 & & 21 & 32 & \\
\hline$>55$ & 43 & 33 & & 43 & 33 & \\
\hline HBsAg & & & 0.796 & & & 0.558 \\
\hline Positive & 51 & 49 & & 51 & 49 & \\
\hline Negative & 14 & 15 & & 13 & 16 & \\
\hline AFP level & & & 0.372 & & & 0.702 \\
\hline$\leq 20 \mathrm{ng} / \mathrm{mL}$ & 30 & 25 & & 26 & 29 & \\
\hline$>20 \mathrm{ng} / \mathrm{mL}$ & 34 & 39 & & 37 & 36 & \\
\hline Tumor size & & & 0.803 & & & 0.803 \\
\hline$\leq 3 \mathrm{~cm}$ & 20 & 21 & & 21 & 20 & \\
\hline$>3 \mathrm{~cm}$ & 45 & 43 & & 43 & 45 & \\
\hline Tumor number & & & 0.329 & & & 0.668 \\
\hline Single & 56 & 51 & & 54 & 53 & \\
\hline Multiple & 9 & 13 & & 10 & 12 & \\
\hline GGT (U/L) & $67(13-888)$ & $83.5(14-2000)$ & 0.182 & $72.5(13-2000)$ & $81(14-1034)$ & 0.156 \\
\hline ALT (U/L) & $36(7-338)$ & $38(12-1198)$ & 0.617 & $42(7-402)$ & $37(12-1198)$ & 0.871 \\
\hline AST (U/L) & $37(18-577)$ & $47.5(17-1474)$ & 0.170 & $38.5(17-577)$ & $42(17-1474)$ & 0.821 \\
\hline $\mathrm{TP}(\mathrm{g} / \mathrm{L})$ & $69.5(56.8-80.6)$ & $67.8(43.4-84.8)$ & 0.171 & $69.1(55.3-80.6)$ & $68.4(43.4-84.8)$ & 0.858 \\
\hline $\mathrm{ALB}(\mathrm{g} / \mathrm{L})$ & $40.3(23.9-48.9)$ & $39.2(21.4-48.6)$ & 0.414 & $40.4(23.8-47.2)$ & $39.4(21.4-48.9)$ & 0.391 \\
\hline
\end{tabular}

AFP: alpha fetoprotein; GGT: gamma-glutamyl transpeptidase; ALT: alanine aminotransferase; AST: aspartic transaminase; TP: total protein; ALB: albumin.

was demonstrated to obtain a fine diagnostic accuracy in distinguishing HCC from LC patients, CHB patients, and healthy controls while the diagnostic potential of serum PTENP1 seems unsatisfactory. The AUC of Linc00152 was greater than those of any other circulating lncRNAs and the currently used tumor marker AFP, indicating remarkable efficacy of Linc00152 in predicting HCC from either benign liver diseases or healthy controls. Nowadays, serum AFP is the most widely used biomarker for HCC diagnosis [4]. However, its sensitivity and specificity are limited. A cohort study of 665 HCC patients carried out by Agopian et al. reported that serum AFP levels do not elevate in $31.3 \%$ of patients with HCC [30]. Therefore, we further investigate whether the combination of these lncRNAs and AFP could improve the diagnostic efficacy of single AFP. Our data revealed that the AUC of all the combinations was greater than single AFP. The best diagnostic performance was obtained in the combined use of linc00152 and AFP with an AUC of 0.906.

Previously, most of the HCC-related lncRNAs focus on single molecule when exploring the potential of lncRNA as novel biomarkers. However, the diagnostic accuracy of any single index is limited. Several studies have already developed several multiple biomarker panels to increase diagnostic accuracy, such as the lncRNA panel established by lncRNALET, PVT1, PANDAR, PTENP1, and linc00963 for the diag- nosis of clear cell renal cell carcinoma [13] and a panel consisting of two lncRNAs (SOX2OT and ANRIL) and three tumor markers (CEA, CYFRA21-1, and SCCA) for the detection of non-small-cell lung cancer [31]. To elevate diagnostic capacity, we identified and combined three markers (linc00152, UCA1, and AFP) by a stepwise selection model, resulting in an AUC of 0.912. The diagnostic sensitivity and specificity of this panel were higher than any single markers. Interestingly, linc00152 and UCA1 have previously been linked with cancers by others. Accumulating evidence support an important role of linc00152 in glioma [32], bladder cancer [33], and HCC [34]. Linc00152 has been demonstrated to promote cell cycle progression in HCC through the miR-193a/b-3p/CCND1 axis [35]. Additionally, elevated serum linc00152 is found in patients with gastric cancer [36], non-small-cell lung cancer [37], and also HCC. The AUC for linc00152 to predicting HCC from healthy control reported by $\mathrm{Li}$ et al. [18] is 0.85 , which is similar to our results. Similarly, frequent upregulation of UCA1 is observed in various cancers, including lung cancer, bladder cancer, gastric cancer, liver cancer, and colorectal cancer [38]. Xiao et al. [39] reported that UCA1 regulates Snail2 expression by effectively sponging miR-203 to promote HCC progression. Moreover, several studies provide strong evidence that serum UCA1 is a potential marker for HCC diagnosis and prognosis [23, 28, 40]. Consistent with our 

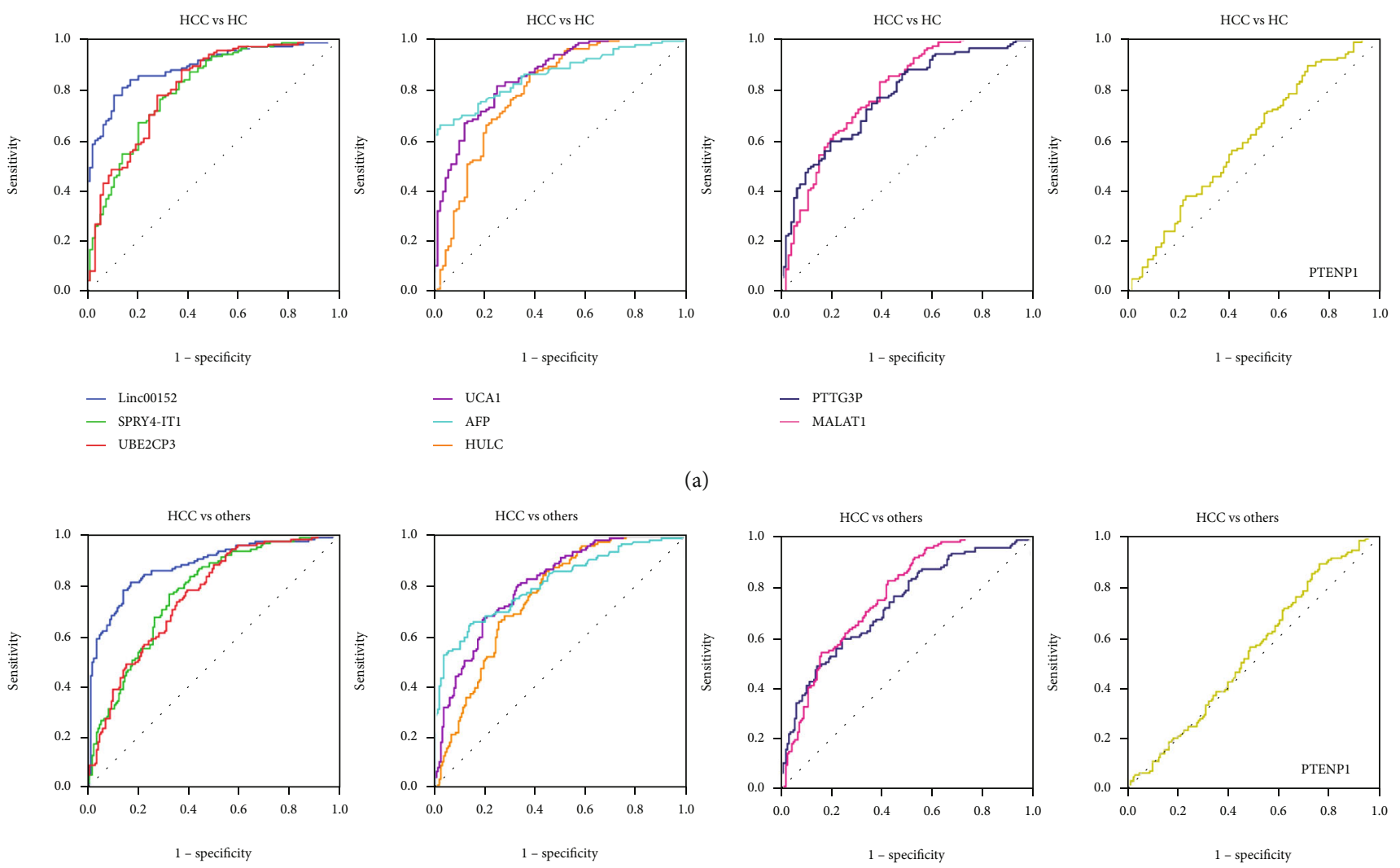

- AFP
- HULC

(a)
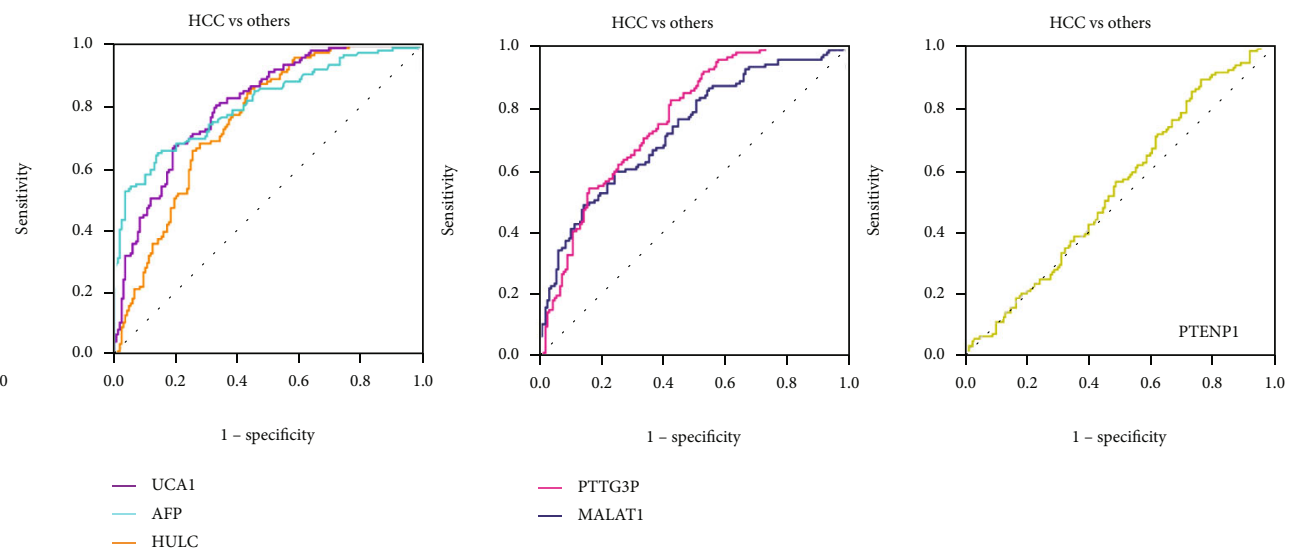

(b)

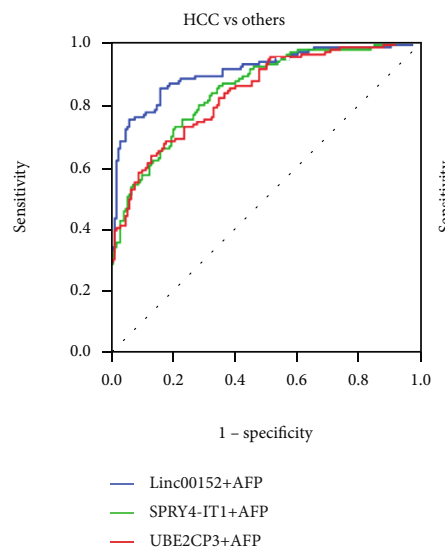

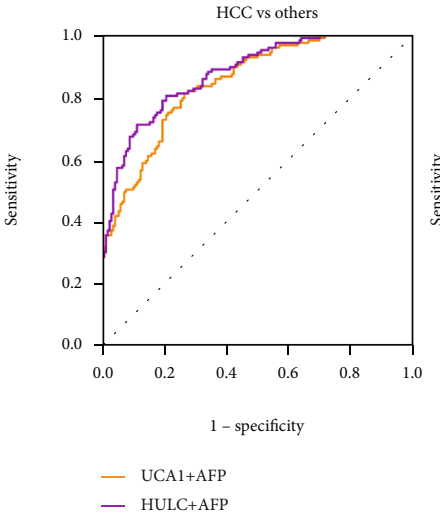

(c)
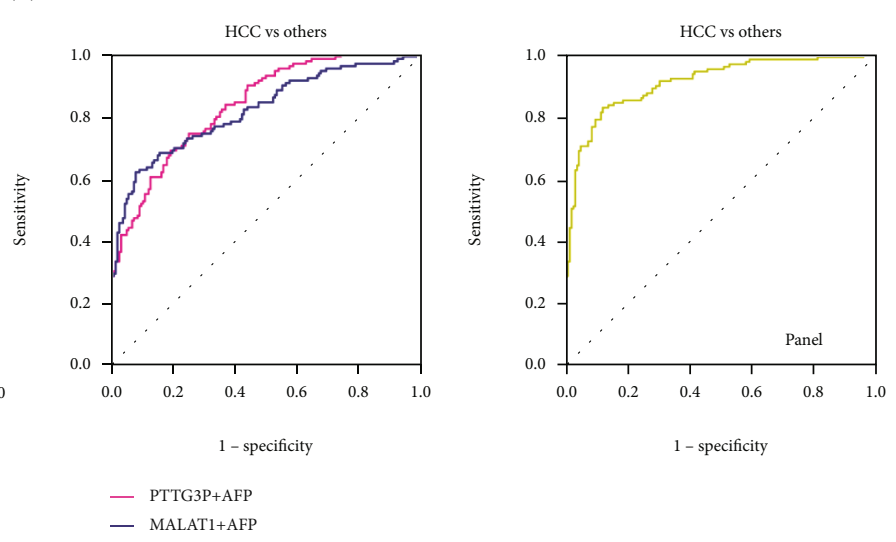

(d)

FIGURE 3: The diagnostic value of serum lncRNAs in patients with HCC. (a) The diagnostic value of HULC, MALAT1, Linc00152, PTENP1, PTTG3P, SPRY4-IT1, UBE2CP3, and UCA1 was evaluated by ROC analysis when patients with HCC were tested against patients with healthy controls. (b) Diagnosis efficacy for HULC, MALAT1, Linc00152, PTENP1, PTTG3P, SPRY4-IT1, UBE2CP3, and UCA1 to distinguish HCC patients from CHB patients, LC patients, and healthy controls. (c) Diagnosis efficacy of the combinations of serum lncRNAs with AFP to discriminate HCC from CHB patients, LC patients, and healthy controls. (d) Diagnosis efficacy of the diagnostic panel established by serum lncRNAs (Linc00512 and UCA1) and AFP.

findings, Zheng et al. [23] revealed that serum UCA1 could differentiate patients with HCC from healthy participants and benign liver diseases. These studies further strengthen our findings that circulating lncRNA linc00152 and UCA1 have high potential to act as HCC biomarkers.
There are a few limitations to our study. First, we did not validate the diagnostic efficacy of the panel in another set. Second, our study was performed at a single center with relatively limited sample size. Thus, further investigations and large-scale multicenter studies are strongly recommended 
TABle 6: Performance of AFP, HULC, MALAT1, Linc00152, PTENP1, PTTG3P, SPRY4-IT1, UBE2CP3, and UCA1 in HC and patients with HCC, CHB, and LC.

\begin{tabular}{|c|c|c|c|c|c|c|}
\hline \multirow{2}{*}{ Method } & \multicolumn{3}{|c|}{ HCC vs. HC } & \multicolumn{3}{|c|}{ HCC vs. others ${ }^{\#}$} \\
\hline & AUC $(95 \%$ CI $)$ & SEN (\%) & SPE (\%) & AUC (95\% CI) & SEN (\%) & SPE (\%) \\
\hline AFP & $0.862(0.815-0.909)$ & 65.9 & 97.8 & $0.811(0.761-0.861)$ & 65.1 & 85.8 \\
\hline HULC & $0.796(0.734-0.858)$ & 86.0 & 62.4 & $0.756(0.702-0.810)$ & 86.0 & 55.6 \\
\hline MALAT1 & $0.768(0.706-0.830)$ & 59.7 & 80.6 & $0.733(0.676-0.790)$ & 59.7 & 75.7 \\
\hline Linc00152 & $0.895(0.854-0.936)$ & 78.3 & 89.2 & $0.877(0.835-0.918)$ & 81.4 & 82.8 \\
\hline PTENP1 & $0.602(0.526-0.678)$ & 89.1 & 29.0 & $0.530(0.465-0.596)$ & 89.1 & 23.1 \\
\hline PTTG3P & $0.785(0.723-0.847)$ & 82.9 & 61.3 & $0.768(0.715-0.820)$ & 82.9 & 57.4 \\
\hline SPRY4-IT1 & $0.808(0.750-0.866)$ & 76.7 & 71.0 & $0.768(0.715-0.821)$ & 76.7 & 67.5 \\
\hline UBE2CP3 & $0.812(0.754-0.870)$ & 88.4 & 62.4 & $0.756(0.702-0.810)$ & 78.3 & 60.4 \\
\hline UCA1 & $0.858(0.810-0.907)$ & 81.4 & 75.3 & $0.809(0.761-0.857)$ & 67.4 & 80.5 \\
\hline
\end{tabular}

\#Others include HC, CHB patients, and LC patients. HCC: hepatocellular carcinoma; HC: healthy control; CHB: chronic hepatitis B; LC: liver cirrhosis; AFP: alpha fetoprotein; AUC: area under curve; CI: confidence interval; SEN: sensitivity; SPE: specificity.

TABLE 7: The performance for the combination of serum lncRNAs and AFP in HC, HCC patients, CHB patients, and LC patients.

\begin{tabular}{lccc}
\hline Method & AUC (95\% CI) & SEN (\%) & SPE (\%) \\
\hline HULC+AFP & $0.848(0.806-0.890)$ & 81.4 & 73.4 \\
MALAT1+AFP & $0.820(0.771-0.870)$ & 62.0 & 92.3 \\
Linc00152+AFP & $0.906(0.870-0.942)$ & 85.3 & 84.0 \\
PTTG3P+AFP & $0.837(0.793-0.881)$ & 69.0 & 81.1 \\
SPRY4-IT1+AFP & $0.847(0.803-0.890)$ & 72.9 & 79.3 \\
UBE2CP3+AFP & $0.837(0.792-0.882)$ & 63.6 & 87.0 \\
UCA1+AFP $_{\text {Panel }}^{\#}$ & $0.878(0.841-0.916)$ & 71.3 & 88.8 \\
\hline
\end{tabular}

HCC: hepatocellular carcinoma; HC: healthy control; CHB: chronic hepatitis B; LC: liver cirrhosis; AFP: alpha fetoprotein; AUC: area under curve; CI: confidence interval; SEN: sensitivity; SPE: specificity. ${ }^{\text {"Panel includes }}$ Linc00152, UCA1, and AFP. Backward stepwise selection was used to determine the diagnostic values of serum IncRNAs for HCC. In this study, the combination of Linc00152, PTENP1, UCA1, and AFP (3-IncRNA panel) was chosen as the strongest panel for diagnostic markers. The other lncRNAs were excluded by a stepwise procedure. The regression equation: $4.528+3.784 \times$ Linc $00152+1.189 \times$ UCA $1+0.005 \times$ AFP.

to fully validate this novel panel. Third, apart from AFP, AFPL3 and PIVKA-II are also common markers for HCC diagnosis. The comparisons between the diagnostic values of these serum lncRNAs and AFPL3 and PIVKA-II for HCC are also recommended for a further study.

\section{Conclusion}

In summary, we established a diagnostic panel consisting of serum lncRNA linc00152, UCA1, and tumor marker AFP with superior sensitivity and specificity to diagnose HCC. This panel might serve as a novel and noninvasive biomarker for HCC diagnosis.

\section{Abbreviations}
AFP:
Alpha fetoprotein
ALB:
Albumin
ALT:
Alanine aminotransferase

AST: $\quad$ Aspartic transaminase

AUC: $\quad$ Area under curve

CHB: $\quad$ Chronic hepatitis B

CI: $\quad$ Confidence interval

GGT: Gamma-glutamyl transpeptidase

HBsAg: $\quad H B V$ surface antigen

HC: Healthy control

HCC: Hepatocellular carcinoma

HULC: Highly upregulated in liver cancer

LC: $\quad$ Liver cirrhosis

lncRNA: Long noncoding RNA

MALAT1: Metastasis-associated lung adenocarcinoma transcript 1

PTENP1: Phosphatase and tensin homolog pseudogene 1

PTTG3P: Pituitary tumor-transforming 3, pseudogene

qRT-PCR: Quantitative real-time polymerase chain reaction

ROC: $\quad$ Receiver operating characteristic curve

SPRY4-IT1: SPRY4 intronic transcript 1

TP: Total protein

UBE2CP3: Ubiquitin conjugating enzyme E2 C pseudogene 3

UCA1: $\quad$ Urothelial carcinoma-associated 1.

\section{Data Availability}

The data that support the findings of this study are not publicly available due to privacy and ethical restrictions but are available from the corresponding author on reasonable request.

\section{Conflicts of Interest}

The authors declare no conflict of interest.

\section{Authors' Contributions}

Jinlan Huang and Yansong Zheng contributed equally to this work. 


\section{Acknowledgments}

This work was supported by grants from the National Natural Science Foundation of China (81802087), the youth project of Fujian Provincial Health and Family Planning Commission (2017-1-47), the Natural Science Foundation of Fujian Province (2018J05130), and the Qihang Project of Fujian Medical University (2017XQ1053). We thank the biomedical laboratory scientists for the excellent technical assistance with handling of blood samples. We also thank the patients for their participation in this study.

\section{References}

[1] R. L. Siegel, K. D. Miller, and A. Jemal, "Cancer statistics, 2018," CA: a Cancer Journal for Clinicians, vol. 68, no. 1, pp. 7-30, 2018.

[2] Y. R. Lee, G. Kim, W. Y. Tak et al., "Circulating exosomal noncoding RNAs as prognostic biomarkers in human hepatocellular carcinoma," International Journal of Cancer, vol. 144, no. 6, pp. 1444-1452, 2019.

[3] A. Villanueva, "Hepatocellular carcinoma," New England Journal of Medicine, vol. 380, no. 15, pp. 1450-1462, 2019.

[4] F. De Stefano, E. Chacon, L. Turcios, F. Marti, and R. Gedaly, "Novel biomarkers in hepatocellular carcinoma," Digestive and Liver Disease, vol. 50, no. 11, pp. 1115-1123, 2018.

[5] R. Chauhan and N. Lahiri, "Tissue- and serum-associated biomarkers of hepatocellular carcinoma," Biomarkers in Cancer, vol. 8s1, pp. BIC.S34413-BIC.S34455, 2016.

[6] J. L. Huang, L. Zheng, Y. W. Hu, and Q. Wang, "Characteristics of long non-coding RNA and its relation to hepatocellular carcinoma," Carcinogenesis, vol. 35, no. 3, pp. 507-514, 2014.

[7] J. Liu, J. Wang, Y. Song et al., "A panel consisting of three novel circulating lncRNAs, is it a predictive tool for gastric cancer?," Journal of Cellular and Molecular Medicine, vol. 22, no. 7, pp. 3605-3613, 2018.

[8] C. Zheng, H. Hao, L. Chen, and J. Shao, "Long noncoding RNAs as novel serum biomarkers for the diagnosis of hepatocellular carcinoma: a systematic review and meta-analysis," Clinical \& Translational Oncology, vol. 19, no. 8, pp. 961968, 2017.

[9] H. Bao and H. Su, "Long noncoding RNAs act as novel biomarkers for hepatocellular carcinoma: progress and prospects," BioMed Research International, vol. 2017, Article ID 6049480, 8 pages, 2017.

[10] A. H. F. El-Tawdi, M. Matboli, H. H. Shehata et al., "Evaluation of circulatory RNA-based biomarker panel in hepatocellular carcinoma," Molecular Diagnosis \& Therapy, vol. 20, no. 3, pp. 265-277, 2016.

[11] S. W. Cao, J. L. Huang, J. Chen et al., "Long non-coding RNA UBE2CP3 promotes tumor metastasis by inducing epithelialmesenchymal transition in hepatocellular carcinoma," Oncotarget, vol. 8, no. 39, pp. 65370-65385, 2017.

[12] L. Dong, P. Qi, M. D. Xu et al., "Circulating CUDR, LSINCT-5 and PTENP1 long noncoding RNAs in sera distinguish patients with gastric cancer from healthy controls," International Journal of Cancer, vol. 137, no. 5, pp. 1128-1135, 2015.

[13] Y. Wu, Y.-Q. Wang, W.-W. Weng et al., “A serum-circulating long noncoding RNA signature can discriminate between patients with clear cell renal cell carcinoma and healthy controls," Oncogenesis, vol. 5, no. 2, p. e192, 2016.
[14] C. L. Chen, Y. W. Tseng, J. C. Wu et al., "Suppression of hepatocellular carcinoma by baculovirus-mediated expression of long non-coding RNA PTENP1 and microRNA regulation," Biomaterials, vol. 44, pp. 71-81, 2015.

[15] J. L. Huang, S. W. Cao, Q. S. Ou et al., "The long non-coding RNA PTTG3P promotes cell growth and metastasis via upregulating PTTG1 and activating PI3K/AKT signaling in hepatocellular carcinoma," Molecular Cancer, vol. 17, no. 1, p. 93, 2018.

[16] I. A. Zaporozhchenko, A. A. Ponomaryova, E. Y. Rykova, and P. P. Laktionov, "The potential of circulating cell-free RNA as a cancer biomarker: challenges and opportunities," Expert Review of Molecular Diagnostics, vol. 18, no. 2, pp. 133-145, 2017.

[17] N. Jiang, J. Pan, S. Fang et al., "Liquid biopsy: circulating exosomal long noncoding RNAs in cancer," Clinica Chimica Acta, vol. 495, pp. 331-337, 2019.

[18] J. Li, X. Wang, J. Tang et al., "HULC and Linc00152 act as novel biomarkers in predicting diagnosis of hepatocellular carcinoma," Cellular Physiology and Biochemistry, vol. 37, no. 2, pp. 687-696, 2015.

[19] P. Malakar, I. Stein, A. Saragovi et al., "Long noncoding RNA MALAT1 regulates cancer glucose metabolism by enhancing mTOR-mediated translation of TCF7L2," Cancer Research, vol. 79, no. 10, pp. 2480-2493, 2019.

[20] L. T. Qin, R. X. Tang, P. Lin et al., "Biological function of UCA1 in hepatocellular carcinoma and its clinical significance: investigation with in vitro and meta-analysis," Pathology, Research and Practice, vol. 214, no. 9, pp. 1260-1272, 2018.

[21] W. Jing, S. Gao, M. Zhu et al., "Potential diagnostic value of lncRNA SPRY4-IT1 in hepatocellular carcinoma," Oncology Reports, vol. 36, no. 2, pp. 1085-1092, 2016.

[22] E. A. Toraih, A. Ellawindy, S. Y. Fala et al., "Oncogenic long noncoding RNA MALAT1 and HCV-related hepatocellular carcinoma," Biomedicine \& Pharmacotherapy, vol. 102, pp. 653-669, 2018.

[23] Z. K. Zheng, C. Pang, Y. Yang, Q. Duan, J. Zhang, and W. C. Liu, "Serum long noncoding RNA urothelial carcinomaassociated 1: a novel biomarker for diagnosis and prognosis of hepatocellular carcinoma," The Journal of International Medical Research, vol. 46, no. 1, pp. 348-356, 2018.

[24] W. Wang, X. He, Z. Zheng et al., "Serum HOTAIR as a novel diagnostic biomarker for esophageal squamous cell carcinoma," Molecular Cancer, vol. 16, no. 1, p. 75, 2017.

[25] H. Konishi, D. Ichikawa, Y. Yamamoto et al., "Plasma level of metastasis-associated lung adenocarcinoma transcript 1 is associated with liver damage and predicts development of hepatocellular carcinoma," Cancer Science, vol. 107, no. 2, pp. 149-154, 2016.

[26] J. G. Yang, X. F. He, B. Huang, H. A. Zhang, and Y. K. He, "Rule of changes in serum GGT levels and GGT/ALT and AST/ALT ratios in primary hepatic carcinoma patients with different AFP levels," Cancer Biomarkers, vol. 21, no. 4, pp. 743-746, 2018.

[27] P. Sun, Y. Li, L. Chang, and X. Tian, "Prognostic and clinicopathological significance of gamma-glutamyltransferase in patients with hepatocellular carcinoma," Medicine, vol. 98, no. 19, p. e15603, 2019.

[28] M. M. Kamel, M. Matboli, M. Sallam, I. F. Montasser, A. S. Saad, and A. H. F. El-Tawdi, "Investigation of long noncoding RNAs expression profile as potential serum biomarkers in 
patients with hepatocellular carcinoma," Translational Research, vol. 168, pp. 134-145, 2016.

[29] S. J. Cho, J. H. Yoon, S. S. Hwang, and H. S. Lee, "Do young hepatocellular carcinoma patients with relatively good liver function have poorer outcomes than elderly patients?" Journal of Gastroenterology and Hepatology, vol. 22, no. 8, pp. 12261231, 2007.

[30] V. G. Agopian, M. P. Harlander-Locke, D. Markovic et al., "Evaluation of patients with hepatocellular carcinomas that do not produce $\alpha$-fetoprotein," JAMA Surgery, vol. 152, no. 1, pp. 55-64, 2017.

[31] Y. Xie, Y. Zhang, L. Du et al., "Circulating long noncoding RNA act as potential novel biomarkers for diagnosis and prognosis of non-small cell lung cancer," Molecular Oncology, vol. 12, no. 5, pp. 648-658, 2018.

[32] M. Yu, Y. Xue, J. Zheng et al., "Linc00152 promotes malignant progression of glioma stem cells by regulating miR-103a3p/FEZF1/CDC25A pathway," Molecular Cancer, vol. 16, no. 1, p. $110,2017$.

[33] Q. Cai, Z. Wang, S. Wang et al., "Long non-coding RNA LINC00152 promotes gallbladder cancer metastasis and epithelial-mesenchymal transition by regulating HIF- $1 \alpha$ via miR-138," Open Biology, vol. 7, no. 1, p. 160247, 2017.

[34] X. Deng, X. f. Zhao, X. q. Liang, R. Chen, Y. f. Pan, and J. Liang, "Linc00152 promotes cancer progression in hepatitis B virusassociated hepatocellular carcinoma," Biomedicine \& Pharmacotherapy, vol. 90, pp. 100-108, 2017.

[35] P. Ma, H. Wang, J. Sun et al., "LINC00152 promotes cell cycle progression in hepatocellular carcinoma via miR-193a/b3p/CCND1 axis," Cell Cycle, vol. 17, no. 8, pp. 974-984, 2018.

[36] T. Yang, H. Zeng, W. Chen et al., "Helicobacter pylori infection, H19 and LINC00152 expression in serum and risk of gastric cancer in a Chinese population," Cancer Epidemiology, vol. 44, pp. 147-153, 2016.

[37] N. Li, X. B. Feng, Q. Tan et al., "Identification of circulating long noncoding RNA Linc00152 as a novel biomarker for diagnosis and monitoring of non-small-cell lung cancer," Disease Markers, vol. 2017, Article ID 7439698, 8 pages, 2017.

[38] A. Bhan, M. Soleimani, and S. S. Mandal, "Long noncoding RNA and Cancer: a new paradigm," Cancer Research, vol. 77, no. 15, pp. 3965-3981, 2017.

[39] J. N. Xiao, T. H. Yan, R. M. Yu et al., "Long non-coding RNA UCA1 regulates the expression of Snail2 by miR-203 to promote hepatocellular carcinoma progression," Journal of Cancer Research and Clinical Oncology, vol. 143, no. 6, pp. 981990, 2017.

[40] A. H. F. El-Tawdi, M. Matboli, S. El-Nakeep, A. E. M. Azazy, and O. Abdel-Rahman, "Association of long noncoding RNA and c-JUN expression in hepatocellular carcinoma," Expert Review of Gastroenterology \& Hepatology, vol. 10, no. 7, pp. 869-877, 2016. 